\title{
Influenza A virus nucleoprotein induces apoptosis in human airway epithelial cells: implications of a novel interaction between nucleoprotein and host protein Clusterin
}

\author{
S Tripathi ${ }^{1}$, J Batra ${ }^{1}$, W Cao ${ }^{2}$, K Sharma ${ }^{1}$, JR Patel ${ }^{2}$, P Ranjan ${ }^{2}$, A Kumar ${ }^{2}$, JM Katz $^{2}$, NJ Cox ${ }^{2}$, RB Lal ${ }^{2}$, S Sambhara ${ }^{2}$ and SK Lal ${ }^{\star, 1}$
}

Apoptosis induction is an antiviral host response, however, influenza A virus (IAV) infection promotes host cell death. The nucleoprotein (NP) of IAV is known to contribute to viral pathogenesis, but its role in virus-induced host cell death was hitherto unknown. We observed that NP contributes to IAV infection induced cell death and heterologous expression of NP alone can induce apoptosis in human airway epithelial cells. The apoptotic effect of IAV NP was significant when compared with other known proapoptotic proteins of IAV. The cell death induced by IAV NP was executed through the intrinsic apoptosis pathway. We screened host cellular factors for those that may be targeted by NP for inducing apoptosis and identified human antiapoptotic protein Clusterin (CLU) as a novel interacting partner. The interaction between IAV NP and CLU was highly conserved and mediated through $\beta$-chain of the CLU protein. Also CLU was found to interact specifically with IAV NP and not with any other known apoptosis modulatory protein of IAV. CLU prevents induction of the intrinsic apoptosis pathway by binding to Bax and inhibiting its movement into the mitochondria. We found that the expression of IAV NP reduced the association between CLU and Bax in mammalian cells. Further, we observed that CLU overexpression attenuated NP-induced cell death and had a negative effect on IAV replication. Collectively, these findings indicate a new function for IAV NP in inducing host cell death and suggest a role for the host antiapoptotic protein CLU in this process.

Cell Death and Disease (2013) 4, e562; doi:10.1038/cddis.2013.89; published online 28 March 2013

Subject Category: Immunity

Influenza A viruses (IAVs) cause frequent epidemics and occasional pandemics, and are one of the leading causes of human morbidity worldwide. A peculiar feature of the IAVs is the induction of massive host cell death in cell culture and animal models. ${ }^{1}$ Apoptosis in general is considered as a host strategy to limit the virus replication. ${ }^{2}$ Paradoxically, host cell death induction is essential for efficient replication of the IAVs and it is closely linked to IAV virulence and pathogenesis. ${ }^{1,3}$ Many IAV proteins such as PB1F2, NS1, M1, M2 and NA are known to modulate the host cell death, invariably by targeting cellular factors. $^{4-10}$ IAV nucleoprotein (NP) has also been implicated in viral pathogenesis and host range determination; ${ }^{11,12}$ however, there has been no report of its direct role in influenza virus-induced cell death. IAV NP is a 498 amino acid protein encoded by segment 5 of the viral RNA genome. ${ }^{13}$ It is one of the most abundant viral proteins and its primary function is to encapsidate the viral genome. ${ }^{13,14}$ It is known to interact with several viral proteins such as PB1, PB2, M1, NS1 and NP itself. ${ }^{14-17}$ It also interacts with a number of host cellular factors including nuclear import receptor $\alpha$ importin, nuclear export receptor CRM1, cytoskeletal element $F$ actin and DEAD-box helicase BAT1/UAP56. ${ }^{12,18-20}$ All these known interactions of IAV NP have been implicated in the intracellular trafficking of the viral genome, viral RNA replication and virus assembly. ${ }^{13}$

In the present study, we have identified a new role for IAV NP in host cell death induction. We show that expression of NP induces apoptosis in host cells. Moreover, we also show that siRNA-mediated knockdown of NP mitigates IAV-induced cell death. The apoptotic effect of IAV NP and its contribution to IAV-induced cell death was significant when compared with other apoptosis modulatory proteins of IAV such as NS1, M1 and M2. Viral proteins often modulate the host apoptotic responses through their interactions with cellular factors. ${ }^{21}$ In a screen to identify cellular interactors of IAV NP, we found a novel and highly conserved interaction between IAV NP and host protein Clusterin (CLU). CLU is ubiquitously expressed, highly conserved and has a regulatory role in cell survival, cell cycle, cell-cell adhesion, cell signaling and protein folding. ${ }^{22}$ CLU prevents cell death by binding to Bax and inhibiting its movement into the mitochondria under stress conditions. ${ }^{23}$ We found that IAV NP weakens the association between CLU

\footnotetext{
${ }^{1}$ Virology Group, International Centre for Genetic Engineering and Biotechnology, New Delhi, India and ${ }^{2}$ Influenza Division, National Center for Immunization and Respiratory Diseases, Centers for Disease Control and Prevention, Atlanta, GA, USA

${ }^{*}$ Corresponding author: SK Lal, Virology Group, International Centre for Genetic Engineering and Biotechnology, New Delhi 110067, India. Tel: +91 11 26742357/1358/ 1361; Fax: +91 11 26742316; E-mail: sunillal@icgeb.res.in

Keywords: influenza; nucleoprotein; apoptosis; clusterin; intrinsic pathway

Abbreviations: IAV, Influenza A virus; NP, nucleoprotein; CLU, Clusterin; Cyt c, Cytochrome C; Casp 3, Caspase 3; CDC, Centers for Disease control; MOI, multiplicity of infection

Received 03.8.12; revised 04.2.13; accepted 11.2.13; Edited by G Ciliberto
} 
and Bax, and CLU overexpression mitigates cell death induced by NP expression or IAV infection. Conversely, siRNA-mediated knockdown of CLU enhanced the apoptosis induced by NP. Taken together, our results suggest a new role for IAV NP in host cell death induction, which is mediated by its interaction with the host antiapoptotic factor CLU.

\section{Results}

Apoptosis induction in IAV-infected cells correlates with NP expression and knockdown of NP reduces IAV-induced cell death. Several studies have suggested a role of IAV NP in viral pathogenesis, however, none have addressed its role in IAV-induced cell death. Thus, we investigated the role of NP in IAV-induced apoptosis. In a time-course study, we examined progression of host cell death by Annexin $\mathrm{V}$ staining and expression of IAV proteins by western blotting in IAV-infected A549 cells. For this experiment, we used A/California/2009(H1N1) strains because it doesn't have a functional PB1F2 protein, ${ }^{24}$ which is an important proapoptotic factor of IAV. ${ }^{4}$ Apoptosis induction was detected as early as $4 \mathrm{~h}$ postinfection and increased until $24 \mathrm{~h}$ (Figure 1a). Expression of the NP protein appeared at $4 \mathrm{~h}$ postinfection (Figure $1 \mathrm{~b}$, panel 1) and its expression kinetics correlated well with the increase in host cell death. Expression of NS1 and M1 proteins appeared at the $8 \mathrm{~h}$ time point (Figure $1 \mathrm{~b}$, panel 2,3). Expression of $\mathrm{M} 2$ peaked towards the end of IAV replication cycle at $24 \mathrm{~h}$ postinfection (Figure 1b, panel 4). Considering the timecourse expression pattern of all the viral proteins, NP seemed to be involved in host cell death induction, specifically between the period of $4-12 \mathrm{~h}$ postinfection. To ascertain the role of NP and compare its contribution in IAVinduced cell death, we inhibited the expression of NP, M1, $\mathrm{M} 2$ and NS1 proteins using siRNAs specific against these genes of A/California/08/2009(H1N1) IAV. The efficacy of the siRNA pools was confirmed in IAV-infected A549 cells (Supplementary Figure. S1A, B, C and D). We observed a significant reduction of cell death in IAV-infected A549 cells in the case of NP knockdown (Figure 1c). Similar effect was

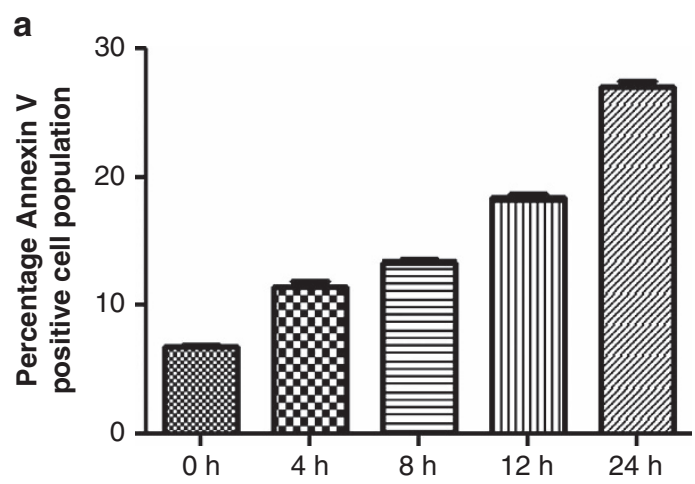

b
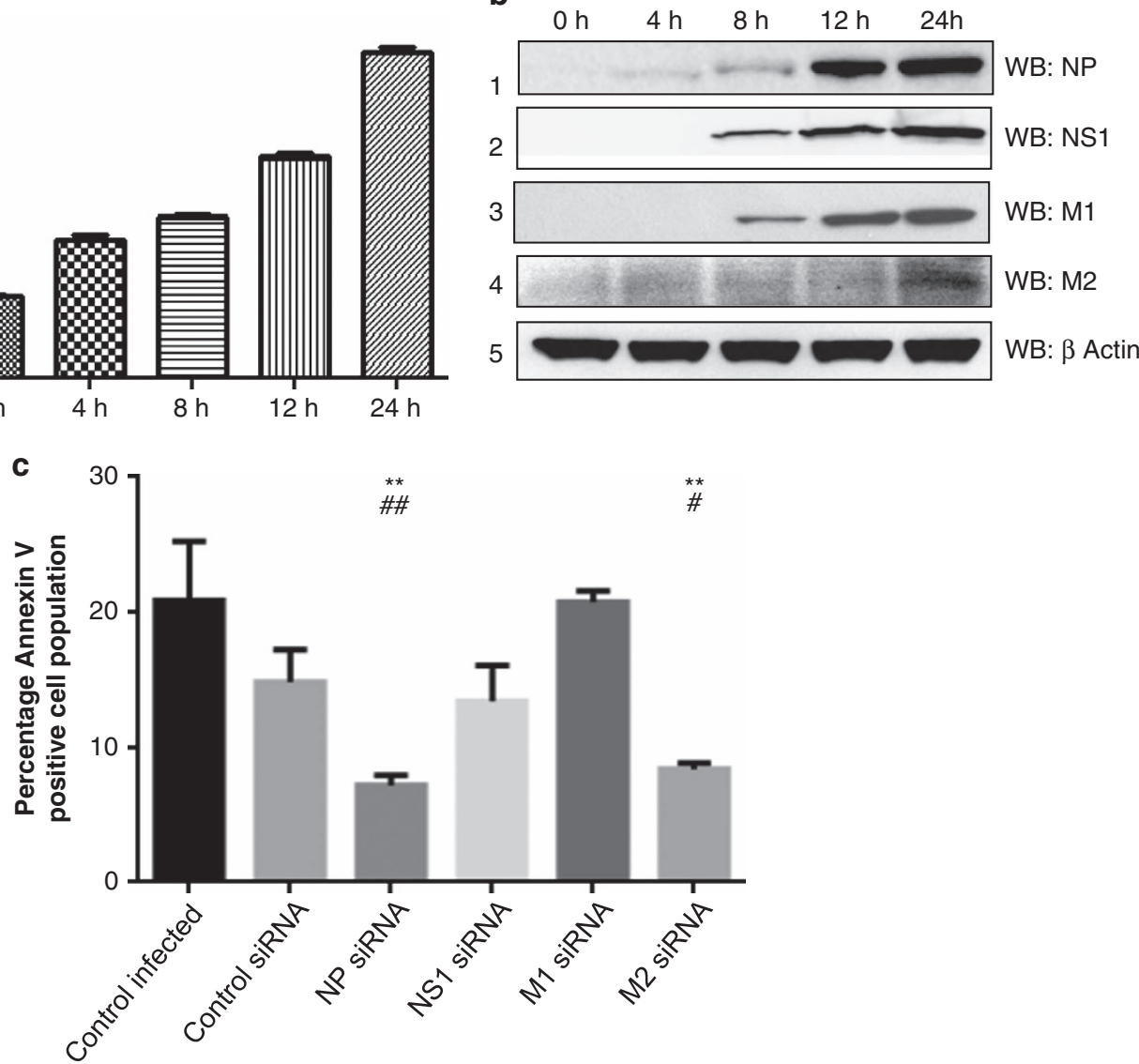

Figure 1 IAV NP contributes to IAV-induced host cell death. (a) A549 cells were infected with A/California/08/2009(H1N1) IAV at an multiplicity of infection (MOI) of 0.2. Cells were harvested at different time intervals, stained with Annexin V PE and subjected to flow cytometry. The percentage of Annexin V-positive cell population is plotted on the graph, which shows means \pm S.D. from one representative experiment $(n=3)$ of at least three independent experiments. (b) From the above mentioned time points, cell lysates were subjected to western blotting using antibodies against NP (rabbit polyclonal: ab 22285), NS1, M1, M2 and $\beta$-actin proteins shown in panels 1, 2, 3, 4 and 5, respectively. (c) A549 cells were transfected with siRNA pool against NP, NS1, M1, M2 gene of A/California/2009(IAV) IAV or control nontargeting siRNA. Six hours post-transfection, cells were infected with respective IAV at $0.2 \mathrm{MOI}$. Cells $12 \mathrm{~h}$ postinfection, stained with Annexin V PE and subjected to flow cytometry. The percentage of Annexin V-positive cell population was plotted on the graph. Data show mean \pm S.D. from one representative experiment $(n=3)$ of at least three independent experiments. Statistical significance was determined using Student's t-test. ${ }^{*} \#<0.05 ;{ }^{* *, \# \#} P<0.01,{ }^{*}$ and ${ }^{\#}$ indicate $P$ values compared to control infected and control siRNA samples respectively 
seen upon M2 knockdown, however, NS1 and M1 knockdown did not alter the IAV-induced cell death (Figure 1c). Taken together, our data indicate that NP contributes to IAV-induced cell death in a significant manner.

Ectopic expression of IAV NP induces apoptosis in human airway epithelial cells. After finding that NP may be involved in IAV infection induced cell death, we checked whether NP expression alone causes apoptosis in host cells. We transfected A549 cells with either a pEGFP-NP plasmid expressing NP protein A/Chicken/Hatay/2004(H5N1) strain, fused to GFP or the control pEGFP plasmid. $48 \mathrm{~h}$ posttransfection, the cells were harvested and stained with
Annexin V PE and 7AAD dyes and examined by flow cytometry. Annexin $\mathrm{V}$ is a marker for early apoptosis, ${ }^{25}$ whereas 7AAD is an indicator of late apoptotic events. ${ }^{26}$ Results showed that cells expressing IAV NP exhibited $\sim$ threefold higher levels of apoptosis as compared with the control (Figures 2a and b). Apoptotic effect of IAV NP was also validated in nontransformed NHBE (normal human bronchial epithelial cells) cells in a similar experiment. A similar apoptotic effect of NP expression was seen in NHBE cells also (Figures $2 c$ and d). These results indicate that the ectopic expression of IAV NP alone can induce apoptosis in human airway epithelial cells. Further, we compared the apoptotic effect of IAV NP with other known a

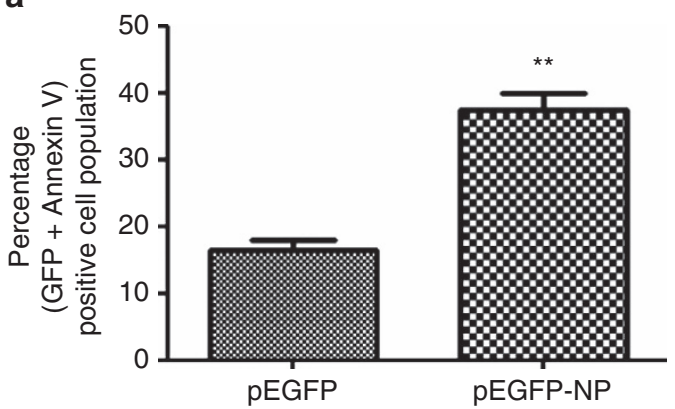

C

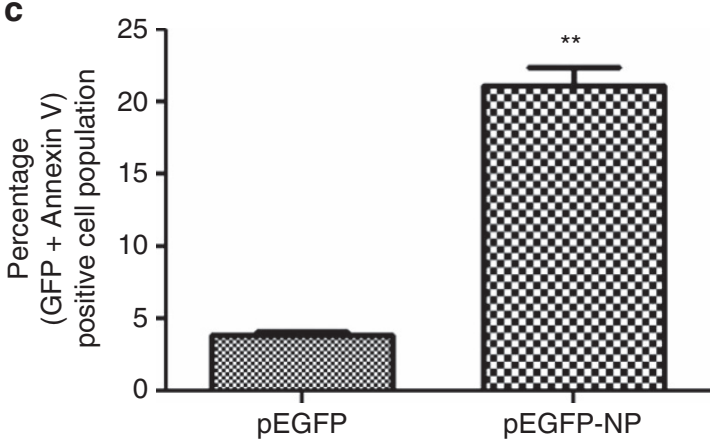

b

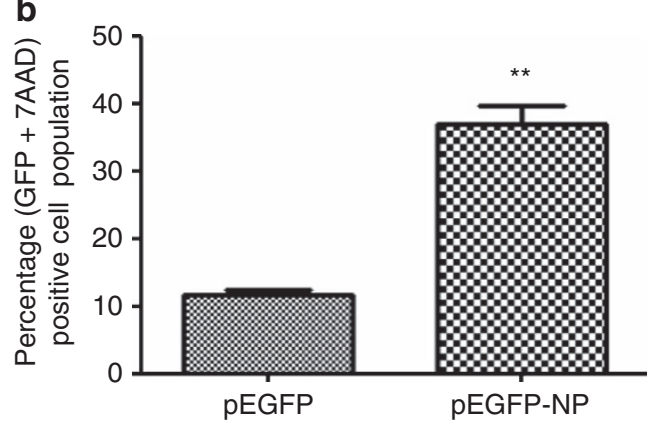

d

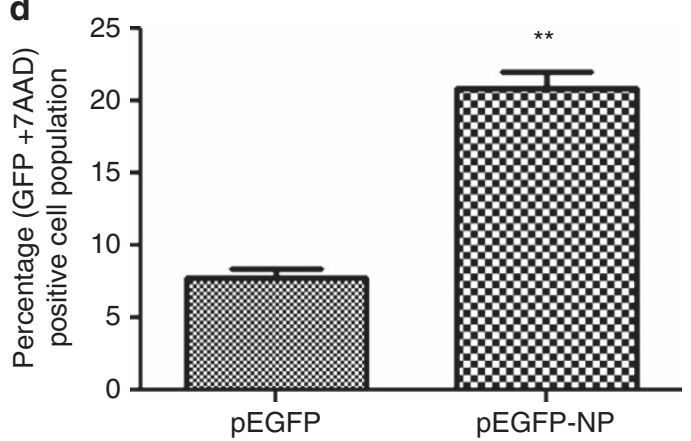

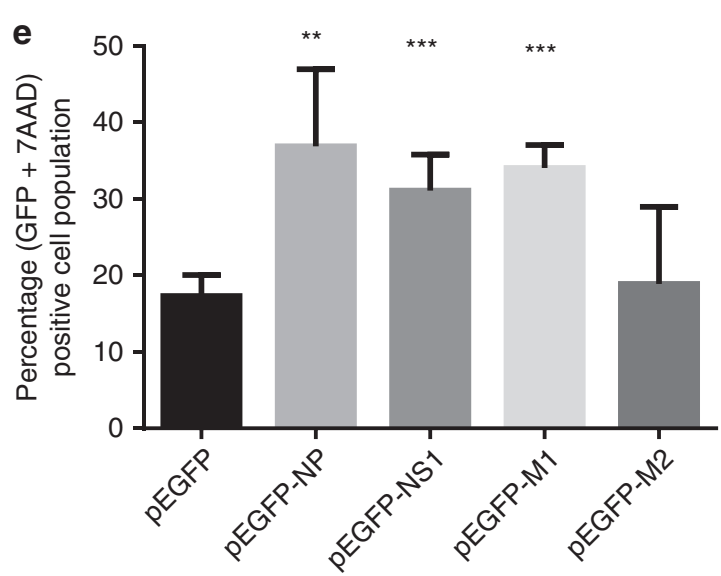

Figure 2 Ectopic expression of IAV NP induces apoptosis in human airway epithelial cells. (a and $\mathbf{b}$ ). A549 cells were transfected with pEGFP-NP plasmid or control pEGFP plasmid. Forty-eight hours post-transfection cells were harvested and stained with Annexin V PE (a) or 7AAD dye (b) and subject to flow cytometry. Annexin V and 7AAD staining in GFP-positive cells was analyzed and plotted as graphs. (c and d). represents similar experiment in NHBE cells. (e). A549 cells were transfected with pEGFP-NP, pEGFP-NS1, pEGFPM1, pEGFP-M2 or control pEGFP plasmid. Forty-eight hours post-transfection cells were harvested and stained with Annexin V PE and subjected to flow cytometry. Annexin V staining in GFP-positive cells was analyzed and plotted as graphs. Data shown in the graphs represents mean \pm S.D. of one representative experiment $(n=3)$ of at least three independent experiments. Statistical significance was determined using student's $t$-test. ${ }^{*} P<0.05$; ${ }^{* \star} P<0.01 ;{ }^{* \star \star} P<0.001$ 
apoptosis regulatory proteins of IAV. To do this, we first cloned NS1, M1 and M2 genes of A/Chicken/Hatay/2004 (H5N1) strain in a pGFPN1 mammalian expression plasmid and confirmed their expression (Supplementary Figure. S2). Then a similar overexpression experiment was conducted as described earlier using these expression plasmids. We observed that NS1 and NP induced similar level, M1 induced lower level and M2 induced significant levels of host cell death (Figure 2e). This suggests that NP expression alone can induce apoptosis in host cells, and it may act through a mechanism independent of other viral factors.

\section{Human antiapoptotic protein CLU interacts with IAV} NP in a conserved and specific manner through CLU $\boldsymbol{\beta}$-chain. To understand the molecular mechanism of IAV NP-induced cell death and possible role of host factors in the same, we conducted a yeast two-hybrid screen using NP gene of A/Chicken/Hatay/2004 (H5N1) strain as bait and a human lung cDNA library as prey. ${ }^{27}$ One of the positive clones was identified by DNA sequencing as the human CLU gene (data not shown). Human CLU protein is translated as an intracellular 449 amino acid protein (sCLU; $\approx 60 \mathrm{kD}$ ), with an N-terminal signal peptide. It targets CLU to ER, where signal peptide is cleaved and SCLU undergoes extensive post-translational modifications to form a heterodimeric glycoprotein, $(\approx 80 \mathrm{kD})$ which is secreted out of the cells. ${ }^{28}$ CLU mRNA also encodes a nuclear form $(\approx 50 \mathrm{kD})$ of CLU ( $\mathrm{nCLU}$ ) by alternative splicing, which is devoid of the signal peptide. $^{29}$ The sCLU is an antiapoptotic protein whereas nCLU is reported to be a proapoptotic factor. ${ }^{23,29}$ The CLU isoform identified as interactor of IAV NP in the yeast twohybrid screen was SCLU and it has been referred as CLU in this report. The NP/CLU interaction was verified by coimmunoprecipitation of A549 cell extracts transfected with plasmids expressing a myc-tagged NP protein of H5N1 IAV and human CLU protein. As shown in Figure 3a, IAV NP immunoprecipitated with overexpressed CLU (lane 2, panel 1) and vice versa (lane 2, panel 2). Furthermore, IAV NP and CLU interaction was verified in A549 cells infected with A/Puerto Rico/8/1934(H1N1) virus by coimmunoprecipitation. Results showed that IAV NP coimmunoprecipitated with endogenous cellular CLU (Figure 3b, lane 1, panel 1) and vice versa (Figure $3 c$, lane 1, panel 2). The molecular weight of CLU protein coimmunoprecipitated with IAV NP was $\approx 60 \mathrm{kD}$, which corresponds to its intracellular form (sCLU). ${ }^{28}$ This interaction was further confirmed in A549 cells infected with several seasonal and pandemic IAV strains. Human CLU protein coimmunoprecipitated consistently with NP protein of all the IAVs tested (Figure 3e, panel 1). The list of various IAV strains used in this experiment is given in the table in Figure 3e. It was observed that NP expression level varied in different IAV strains (Figure 3e, panel 3), this may be attributed to the difference in their replication rates in A549 cells. These results indicate that IAV NP interacts with cellular endogenous CLU in a conserved manner. We also checked the interaction of CLU with M1, M2 and NS1 in IAVinfected cells and found that CLU specifically interacts only with NP (Supplementary Figure S3, lane 2).

Further, we characterized the CLU protein domain responsible for interaction with IAV NP by GST pull-down assay.
The nascent CLU protein is translated with an $\mathrm{N}$-terminal signal peptide followed by a CLU $\beta$-chain and C-terminal CLU $\alpha$-chain. In the mature CLU protein, the signal peptide is cleaved and $\beta$ - and $\alpha$-chains are joined by disulfide linkages. ${ }^{28}$ Therefore, we cloned full-length mature CLU (without signal peptide), CLU $\beta$-chain and CLU $\alpha$-chain proteins in a GST tag bacterial expression vector (Supplementary Figure S4A) and IAV NP was cloned into a His Tag bacterial expression vector (Supplementary Figure S4A). The recombinant proteins were expressed in Escherichia coliand GST pull-down assays were carried out. His-tagged IAV NP was detected in the GST pulldown fraction of full-length CLU and CLU $\beta$-chain (Supplementary Figure S4B, panel 1, lane 1, 2); whereas NP showed no interaction with CLU $\alpha$-chain or GST control (Supplementary Figure S4B, panel 1, lane 3, 4). Overall, the results showed that $\mathrm{CLU} \beta$-chain was responsible and sufficient for interaction with IAV NP.

IAV NP and CLU colocalize during late stages of IAV infection in perinuclear region of the cells. Next, we sought to determine the stage of IAV infection and the intracellular sites where NP and CLU may interact. To this end we checked NP and CLU localization by immunofluorescence microscopy and biochemical fractionation in an IAV infection time-course study. We observed that NP localized in the nucleus at $4 \mathrm{~h}$ postinfection (Figure $4 \mathrm{~b}$ ). At $8 \mathrm{~h}$ and $12 \mathrm{~h}$ time points it moved out to the cytoplasm (Figures $4 c$ and d), as reported elsewhere. ${ }^{13} \mathrm{CLU}$ had diffused nucleocytoplasmic localization in control uninfected cells (Figure 4a), however, in IAV-infected cells, it localized extensively in the perinuclear region (Figures $4 \mathrm{~b}, \mathrm{c}, \mathrm{d}$ ). It was observed that IAV NP and CLU colocalized primarily in the perinuclear region, at later stages of infection (Figures $4 \mathrm{c}$ and $\mathrm{d}$ ). Biochemical fractionation of IAV-infected cells at similar time points showed that NP and CLU accumulated in the membrane fraction between 8 and $12 \mathrm{~h}$ postinfection (Supplementary Figure S5, panel 1, 2, lane 4, 5). The membrane fraction represents primarily the ER/Golgi and mitochondrion of the cells, and the purity of these fractions was checked by specific markers for ER (Calnexin) and cytosol (GAPDH) (Supplementary Figure S5, panel 3, 4). These results indicate that IAV NP and CLU interact during the late stages of infection in the cytoplasm of IAV-infected cells undergoing apoptosis.

IAV NP induces cell death through the intrinsic apoptosis pathway. Considering that CLU inhibits intrinsic apoptosis pathways ${ }^{23}$ and interacts with IAV NP, we checked whether IAV NP-induced cell death is also mediated by the same cell death pathway. Hallmarks of this pathway are the movement of Bax into mitochondria, release of Cytochrome $\mathrm{c}$ (Cyt c) from the mitochondria and subsequent activation of Caspase 3 (Casp 3). ${ }^{30}$ We studied the effect of IAV NP expression on these events by immunofluorescence microscopy. It is known that Bax is present in the nuclei as well as cytoplasm of many cell lines. ${ }^{31}$ Similarly, we observed that Bax localized in the nucleus of control A549 cells (Figure 5a); however, in IAV NP-transfected cells, a substantial amount of Bax was detected in the mitochondrial region (Figure 5b). Furthermore, in control cells, Cyt $\mathrm{c}$ was localized in the 
a

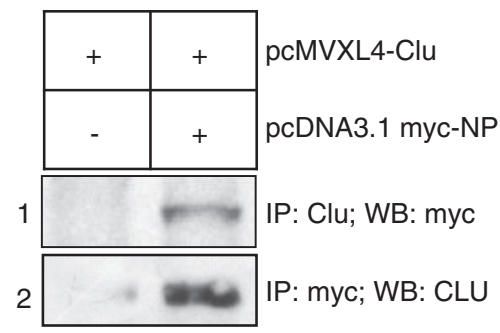

C

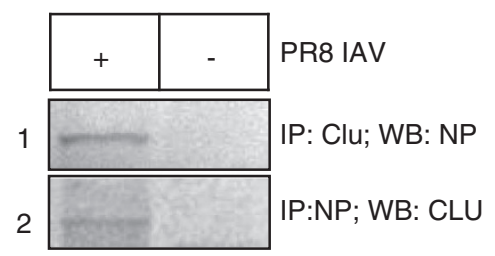

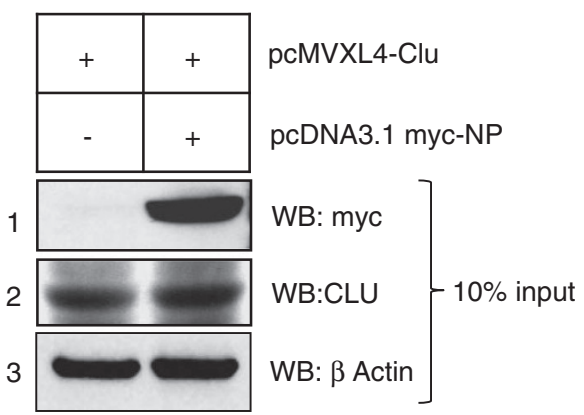

d

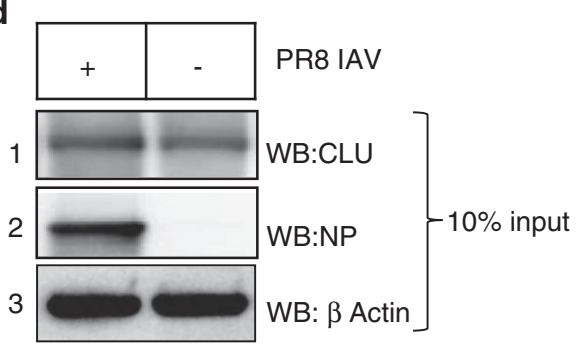

e

\begin{tabular}{|l|l|}
\hline Lane & Influenza A Virus Strain used \\
\hline 1 & Control \\
\hline 2 & A/ Wisconsin/ 67/ 2005 (H3N2) \\
\hline 3 & A/ Solomon Island/ 3/ 2006 (H1N1) \\
\hline 4 & A/ California/ 08/ 2009 (H1N1) \\
\hline 5 & A/ Vietnam/ 1203/ 04 (H5N1) \\
\hline 6 & A/ Hong Kong/ 483/ 97 (H5N1) \\
\hline
\end{tabular}

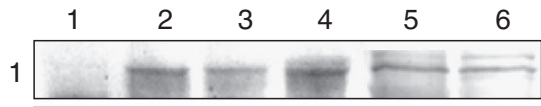

IP: NP; WB: CLU

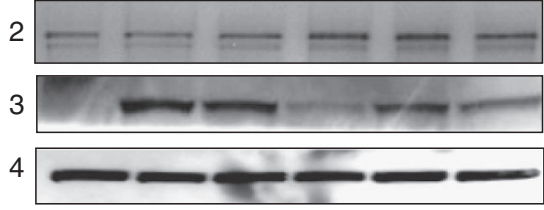

$\left.\begin{array}{l}\text { WB: CLU } \\ \text { WB: NP } \\ \text { WB: } \beta \text { Actin }\end{array}\right\} 10 \%$ input

Figure 3 IAV NP interacts with human CLU protein in human lung epithelial cells. (a) A549 cells were transfected with (pcDNA3.1 myc-NP + pCMV XL4-CLU) plasmids or only with pCMV XL4-CLU plasmids. Cells were harvested $48 \mathrm{~h}$ post-transfection and immunoprecipitation was set up using anti-myc tag and anti-CLU antibodies, followed by western blotting. Lane 2 of panel 1 shows coimmunoprecipitation of myc-tagged NP with CLU and lane 2 of panel 2 shows coimmunoprecipitation of CLU with myc-tagged NP. Lane 1 of panel 1 and 2 represents samples transfected with control plasmid. (b) From the previous experiment $10 \%$ input cell lysate was subjected to western blotting with anti-Myc (panel 1), anti-CLU (panel 2) and anti- $\beta$-actin (panel 3) antibodies. (c) A549 cells were infected with PR8 IAV at $1 \mathrm{MOI}$ and harvested $24 \mathrm{~h}$ postinfection. Immunoprecipitation was set up using NP-specific antibody and CLU-specific antibody. Lane 1 of panel 1 shows coimmunoprecipitation of viral NP with CLU and lane 1 of panel 2 shows coimmunoprecipitation of CLU with NP. Lanes 2 of panel 1 and 2 represent control uninfected samples. (d) From the previous experiment $10 \%$ input cell lysate was subjected to western blotting with anti-CLU (panel 1), anti-NP (panel 2) and anti- $\beta$-actin (panel 3) antibodies (e) A549 cells were infected with different IAV isolates, as indicated in the Table, at $1 \mathrm{MOI}$. Cells were harvested $24 \mathrm{~h}$ postinfection and immunoprecipitation was set up using NP-specific antibody, followed by western blotting. Panel 1 shows coimmunoprecipitation of CLU with NP of different IAV strains. Panels 2, 3 and 4 show expression levels of CLU, NP and $\beta$-actin in the cell lysates used for immunoprecipitation. Lane 1 of all panels shows control uninfected samples. List of the IAV strains used for infection is provided in the table

perinuclear-mitochondrial region, (Supplementary Figure S6A) whereas it acquired a diffused cytoplasmic localization, indicating its release from the mitochondria, in IAV NPexpressing cells (Supplementary Figure S6B). The Cyt c release was also quantified by flow cytometry, and our result showed $\approx$ threefold higher Cyt release from mitochondria in IAV NP-transfected cells as compared with control (Figure 5c). Next we checked the effect of NP expression on Casp 3 activity by flow cytometry. Results from this experiment revealed that IAV NP induced around threefold more Casp 3 activity compared with control (Figure 5d). These results correlated well with the level of apoptosis induction by IAV NP expression, suggesting that NP-induced cell death occurs through the intrinsic apoptosis pathway.
CLU attenuates IAV NP-induced cell death and IAV replication. Further, we investigated whether CLU was involved in IAV NP-induced cell death. We checked the effect of CLU overexpression and CLU knockdown on cell death induction by IAV NP expression or IAV infection. CLU was ectopically expressed in A549 cells, followed by infection with IAV or transfection with IAV NP-expressing plasmid, and cell death was assessed by Annexin $V$ staining. We observed that the overexpression of CLU in A549 cells reduced IAV NP-induced cell death by $\approx 20 \%$ (Figure 6a) as compared with control. In IAV-infected cells also, the CLU overexpression reduced cell death by $\approx 20 \%$ (Figure $6 \mathrm{~b}$ ) as compared with control. The effect of CLU overexpression on virus replication was also checked by detecting NP protein 
a
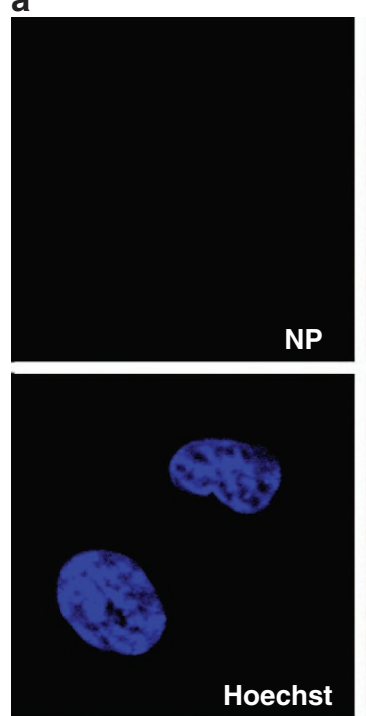

C
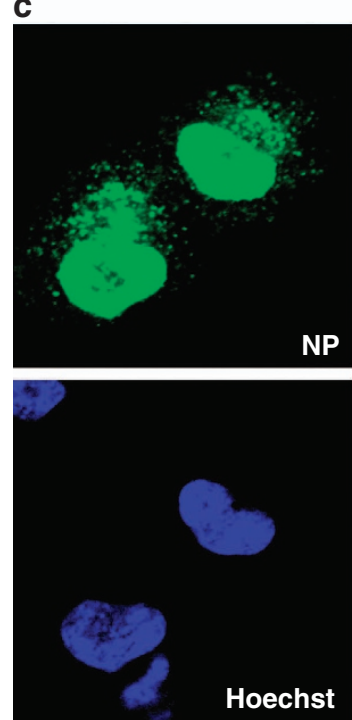
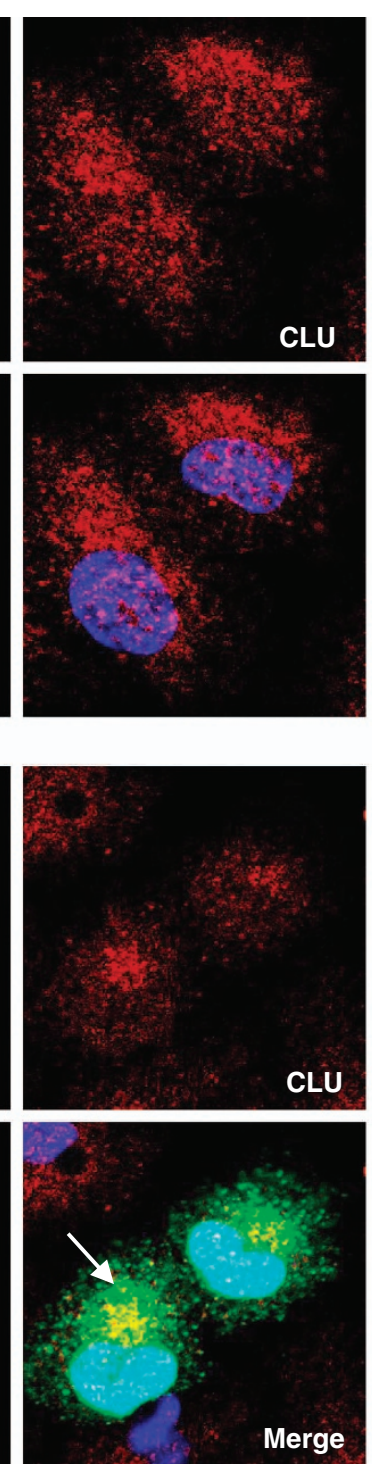

b
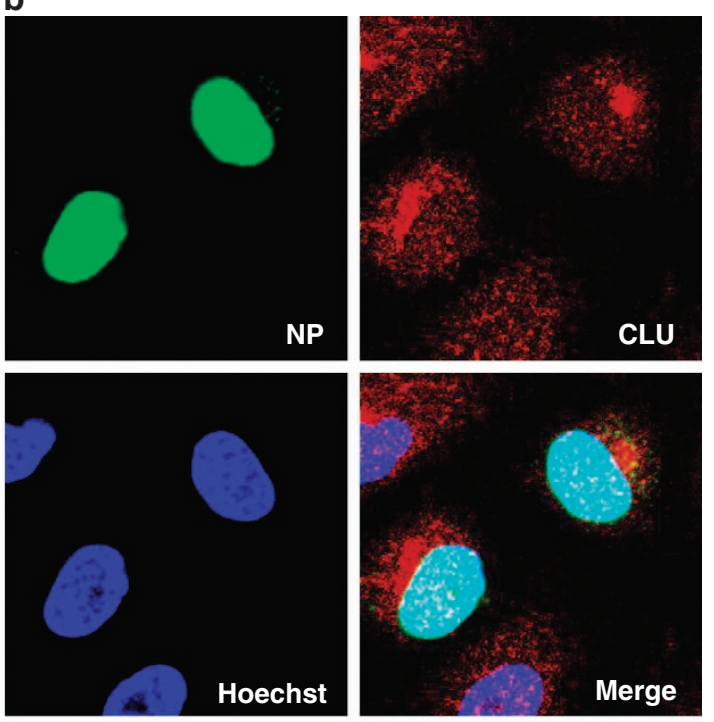

d
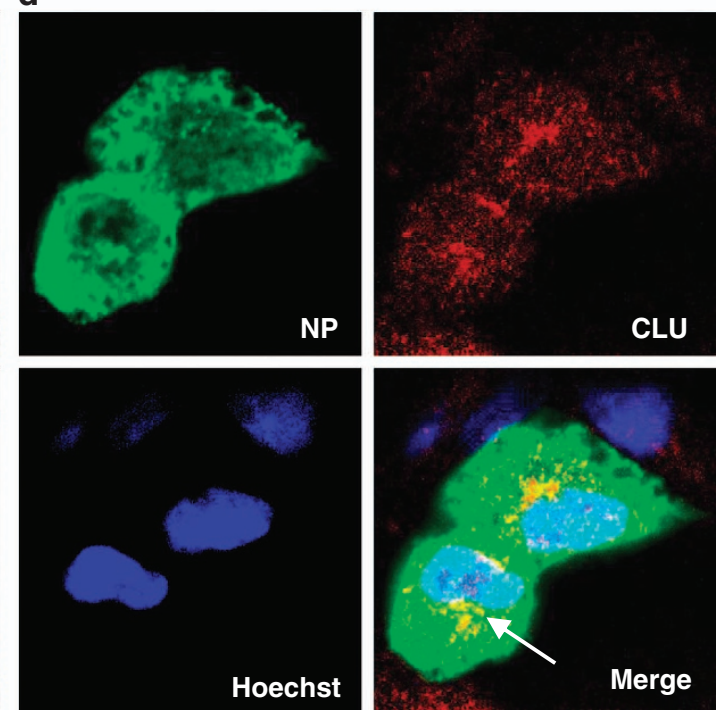

Figure 4 IAV NP and CLU colocalize during late stages of IAV infection in perinuclear region of the cells. (a-d) A549 cells were infected with PR8 IAV at five MOI, cells were fixed at different time points and processed for immunostaining and observed under confocal microscope. NP was stained using anti-NP monoclonal primary antibody and Alexa488 conjugated secondary antibody (green). CLU was stained using CLU-specific primary antibody and Alexa 594 conjugated secondary antibody (red). Nuclei were stained with Hoechst stain. (a) shows control uninfected cells; (b, c and d) show IAV-infected cells at 4, 8 and $12 \mathrm{~h}$ postinfection, respectively. Panels are labeled for their respective staining. Image region showing colocalization is indicated by arrows

expression level and also by plaque assay. CLU overexpression in IAV-infected cells reduced NP protein expression (Figure 6c, panel 3, lane 3) and decreased virus titers by $40 \%$ (Figure $6 \mathrm{~d}$ ). We tested the effect of siRNA-mediated knockdown of CLU on IAV NP-induced apoptosis. We observed that CLU inhibition enhanced cell death by $\approx 30 \%$ as compared with control siRNA-treated control cells in case of both NP plasmid transfection (Figure 6e) and IAV infection (Figure 6f). The efficacy of the siRNA pool used against CLU in this experiment was checked by western blotting, and the results clearly showed knockdown of CLU expression as compared with the control (Supplementary Figure S7A, panel 1, lane 3). As CLU overexpression mitigated, whereas CLU knockdown exacerbated
NP-induced apoptosis, we postulated that CLU is targeted by NP for cell death induction.

IAV NP interferes with CLU-Bax association. As Bax movement to the mitochondria requires its dissociation from CLU, ${ }^{23}$ we checked the effect of IAV NP expression on CLUBax association. For this A549 cells were transfected with IAV NP-expressing plasmid or control plasmid. Two days post-transfection, cells were harvested and immunoprecipitation was carried out with CLU-specific antibody. We detected coimmunoprecipitation of both IAV NP and Bax along with CLU (Figure 7a, panel 1, 2, 3); however, in IAV NP-transfected cells, the amount of Bax coimmunoprecipitated with CLU was reduced (Figure 7a, panel 2, lane 2). This 
a
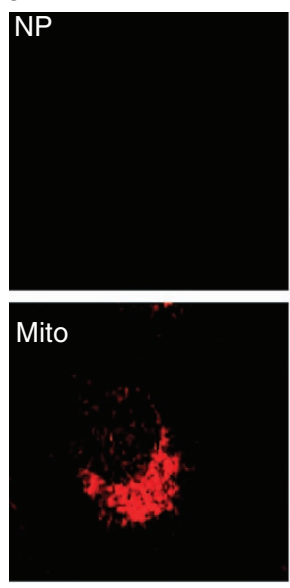

pcDNA3.1 myc b
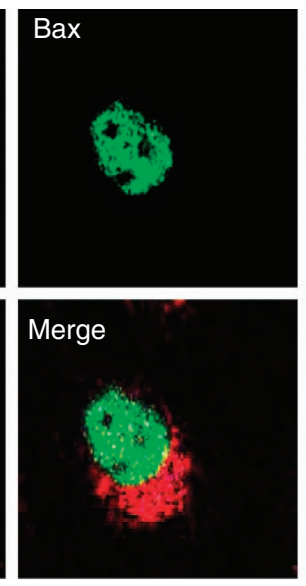

.

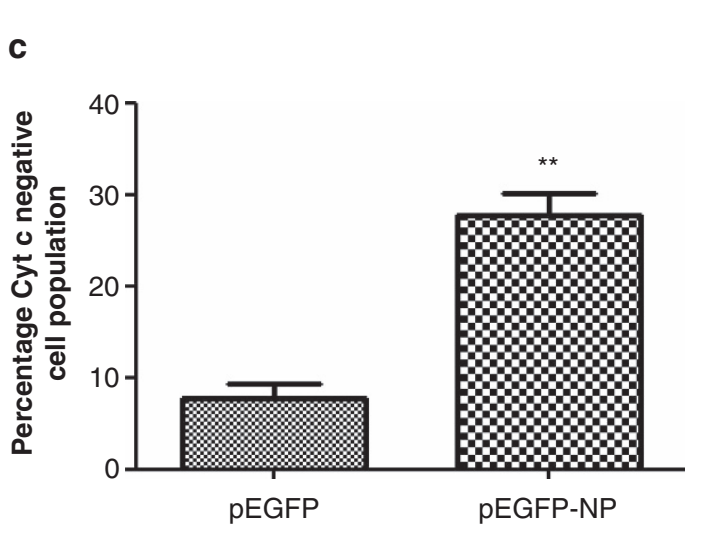

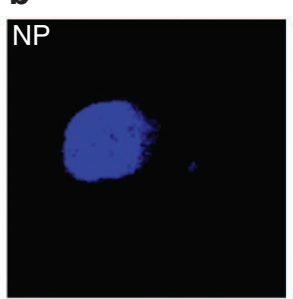
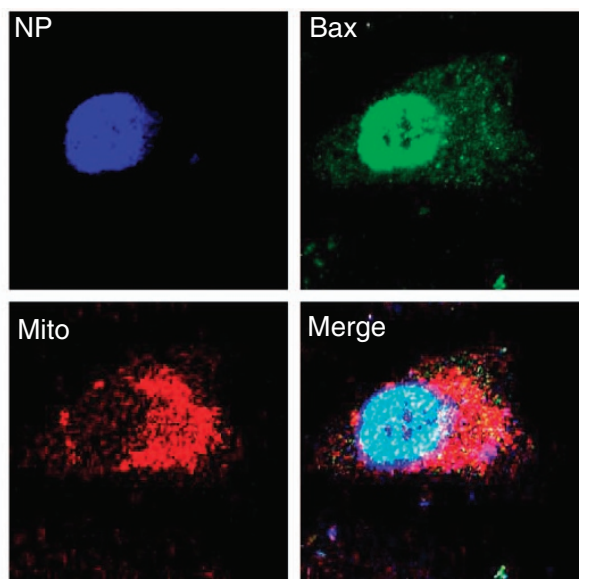

pcDNA3.1 myc-NP

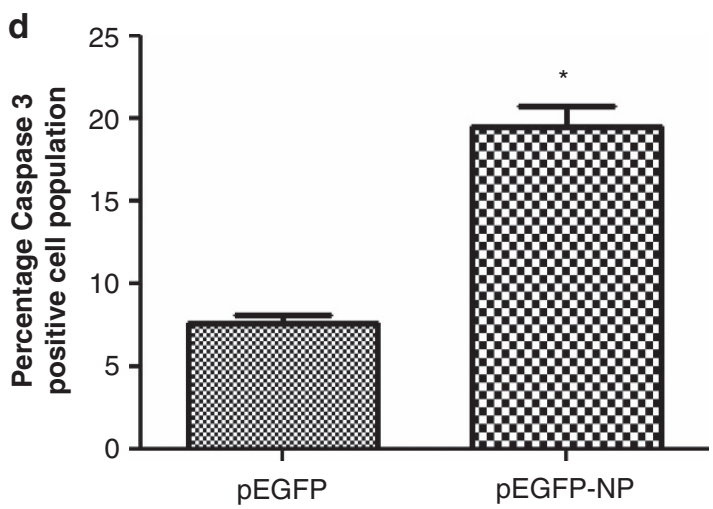

Figure 5 IAV NP-induced cell death involves the mitochondrial apoptosis pathway. (a and b) A549 cells were transfected with pcDNA3.1 myc-NP or control pcDNA3.1 myc plasmid. Forty-eight hours later cells were processed for immunostaining and observed under confocal microscope. NP was stained using anti-myc tag primary antibody and Alexa350 conjugated secondary antibody (blue). Bax was stained using Bax-specific primary antibody and Alexa 488 conjugated secondary antibody (green). Mitochondria were stained with CMXRos Red mitotracker dye. Panels are labeled for their respective staining. (a) shows pcDNA3.1 myc-NP-transfected cells, whereas (b) shows control pcDNA3.1 myc-transfected cells. (c) A549 cells were transfected with pEGFP-NP or control pEGFP plasmid. Forty-eight hours post-transfection cells were stained using FlowCellect Cytochrome $\mathrm{c}$ kit (Millipore), which differentially stains Cyt c present in the mitochondria. The percentage of (GFP positive + Cyt c negative) population is shown in the graph. (d) A549 cells were transfected with pEGFP-NP plasmid or control pEGFP plasmid. Forty-eight hours post-transfection cells were harvested and stained with PE Active Caspase 3 staining kit (BD Biosciences), which differentially stains cleaved form of Casp 3, and subjected to flow cytometry. The percentage of (GFP positive + Casp 3 positive) population is shown in the graph. All graphs represent mean \pm S.D. of one representative experiment $(n=3)$. Statistical significance was determined using Student's t-test. ${ }^{*} P<0.05,{ }^{* *} P<0.01$

clearly indicates that IAV NP interferes with CLU-Bax association, which may result in Bax movement into the mitochondria.

\section{Discussion}

Apoptosis induction by IAVs is a multifactorial process, ${ }^{32}$ as many virus and host factors contribute to it in a complex manner. ${ }^{1}$ In the present study, we show that IAV NP induces host cell death through the mitochondrial apoptosis pathway. We also report a novel interaction between IAV NP and host protein CLU. Our results indicate that CLU has a cytoprotective role in IAV infection, and IAV NP targets CLU to induce cell death. The question, whether apoptosis induction favors IAV or host, is still under debate. ${ }^{1}$ However, there are many reports that suggest that apoptosis induction by IAV facilitates virus replication, dissemination and killing of the host immune effectors cells. ${ }^{32-36}$ Cell death induced by IAV may depend on the host cell type and the stage of infection. ${ }^{37}$

We validated apoptotic phenotype of NP in the human tracheal/bronchial as well as alveolar epithelial cells, which are the primary site of IAV infection and replication in humans. ${ }^{38}$ We compared the apoptotic effect of IAV NP with other IAV proteins and found that NP was a major contributor of IAV-induced cell death. The PB1F2 protein of influenza viruses is also known to induce cell death through the mitochondrial pathway, ${ }^{4}$ therefore we used A/California/ 2009 (H1N1) influenza virus that allowed us to study IAVinduced apoptosis in the absence of PB1F2. ${ }^{24}$ We also observed that although M2 expression did not induce cell death, M2 knockdown in IAV-infected cells mitigated apoptosis. This suggests that $\mathrm{M} 2$ may require additional viral factors to contribute to host cell death. Our results showed that NS1 and M1 proteins promote cell death in overexpression 
a

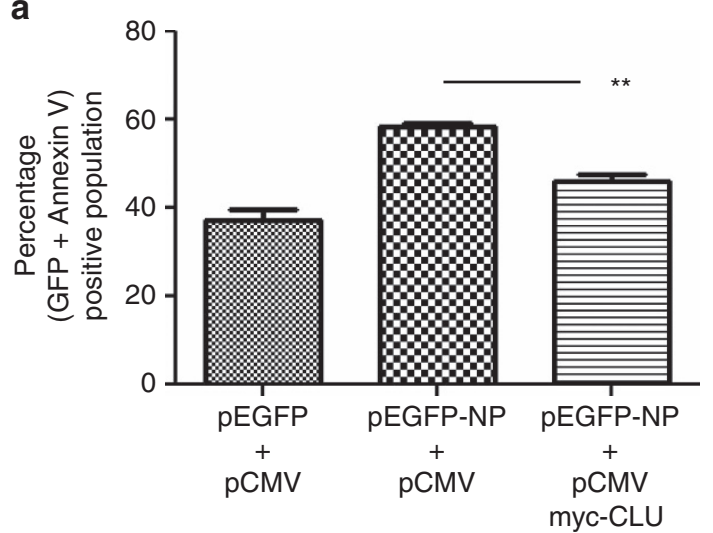

C
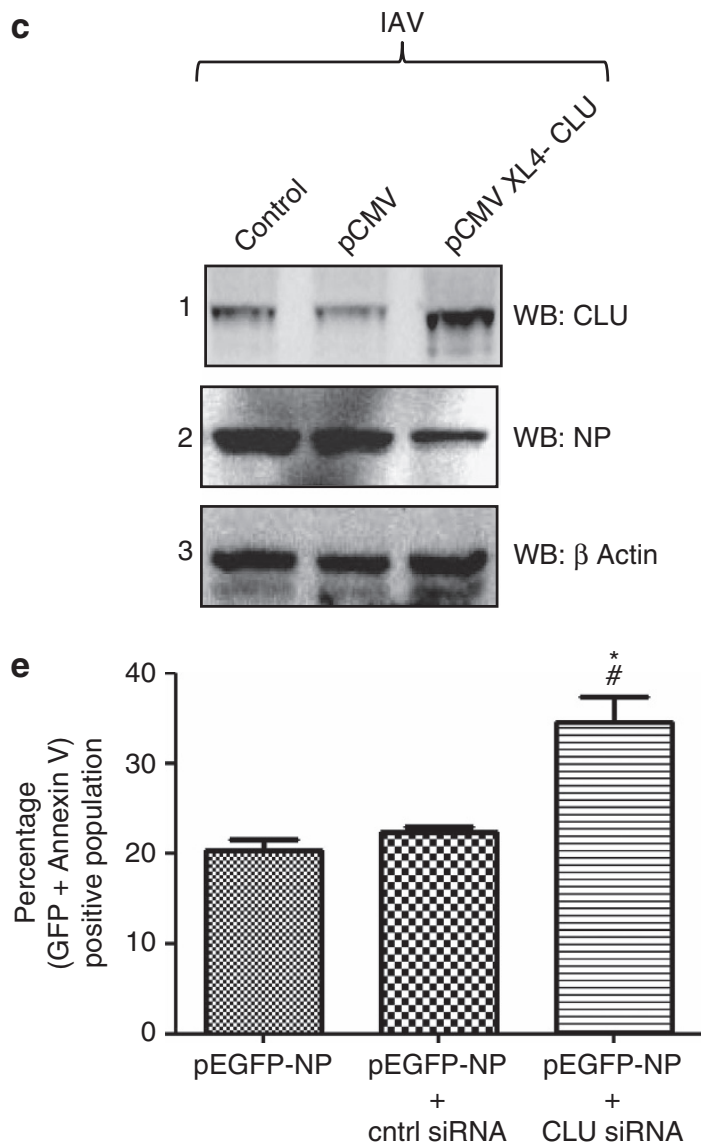

b

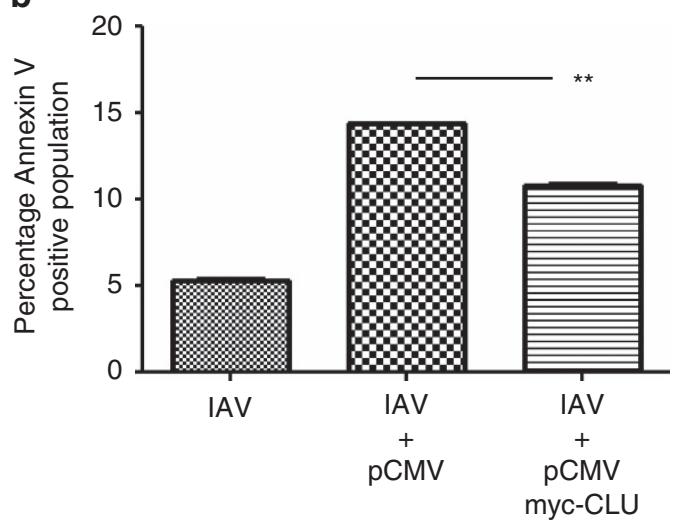

d

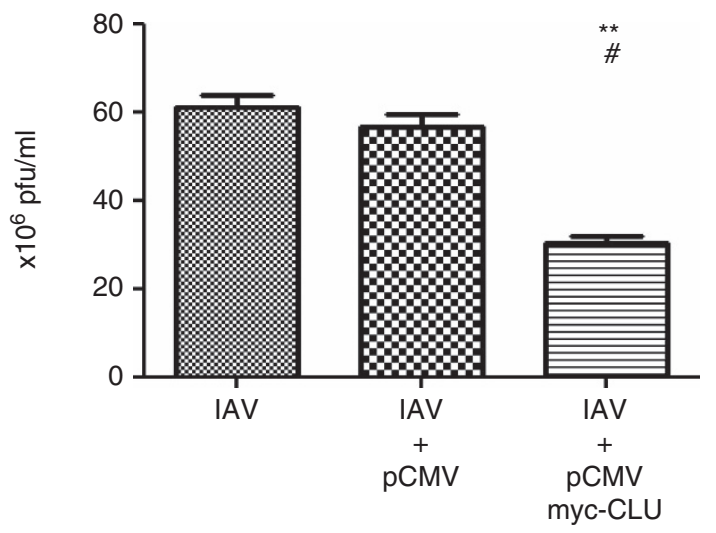

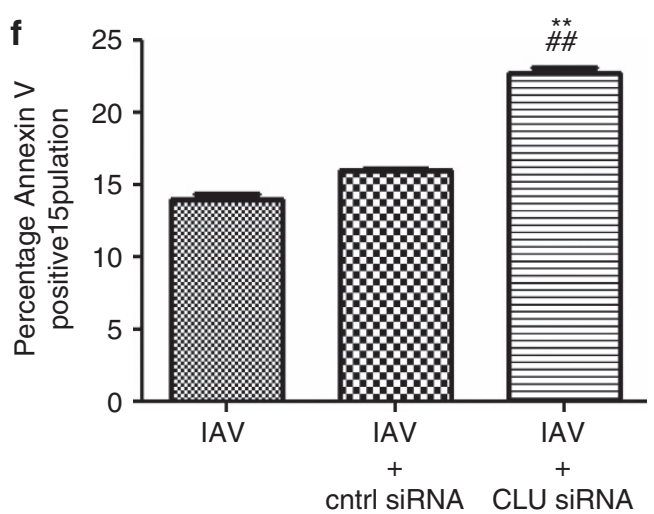

Figure 6 CLU attenuates IAV NP-induced cell death and IAV replication. (a) A549 cells were transfected with CLU-expressing plasmid pCMV XL4-CLU or control plasmid pCMV. Twenty-four hours post-transfection, cells were transfected with pEGFP-NP plasmid or pEGFP control plasmid. Cells were harvested at $24 \mathrm{~h}$ postinfection, stained with Annexin V PE and subjected to flow cytometry. The percentage of (GFP + Annexin V) positive population was plotted on the graph. (b) A549 cells were transfected with CLUexpressing plasmid pCMV XL4-CLU or control plasmid pCMV. Twenty-four hours post-transfection, cells were infected with A/California/08/2009(H1N1) IAV at 0.2 MOI. Cells were harvested at $12 \mathrm{~h}$ postinfection, stained with Annexin V PE and subjected to flow cytometry. The percentage of Annexin V-positive population was plotted on the graph. (c) A549 cells were transfected with CLU-expressing plasmid PCMV XL4-CLU or control plasmid pCMV. Twenty-four hours post-transfection, cells were infected with A/California/08/ 2009(H1N1) IAV at $0.2 \mathrm{MOI}$. Cells were harvested at $24 \mathrm{~h}$ postinfection, and the cell lysates were subject to SDS-PAGE followed by western blotting with antibodies specific to CLU, NP and $\beta$ actin, which are shown in panels 1,2, and 3 respectively. Lane 1 shows control cells, lane 2 shows cells transfected with control plasmid and lane 3 shows cells transfected with CLU-expressing plasmid, all of which are infected with IAV. (d) Culture supernatant from the experiment shown in (c) were harvested and used to set up plaque assay to study virus replication. The $10^{6} \times \mathrm{pfu} / \mathrm{ml}$ was plotted on the graph. (e) A549 cells were transfected with siRNA pool against CLU gene or nontargeting control siRNA. Twenty-four hours post-transfection, cells were transfected with pEGFP-NP plasmid. Twenty-four hours postinfection cells were harvested and stained with Annexin V PE and subjected to flow cytometry. The percentage of (GFP + Annexin V) positive population was plotted on the graph, ${ }^{*}$ and ${ }^{\#}$ indicate $P$ values compared to $p E G F P-N P$ and pEGFPNP + control siRNA samples respectively. (f) A549 cells were transfected with siRNA pool against CLU gene or nontargeting control siRNA. Twenty-four hours post-transfection; cells were infected with A/California/2009(H1N1) IAV at $0.2 \mathrm{MOI}$. Twelve hours postinfection cells were harvested and stained with Annexin V PE and subjected to flow cytometry. The percentage of Annexin V-positive population was plotted on the graph. All graphs represent mean \pm S.D. of one representative experiment $(n=3)$. Statistical significance was determined using Student's $t$-test. ${ }^{*} P<0.05 ;{ }^{* *} P<0.01$, ${ }^{*}$ and ${ }^{\#}$ indicate $P$ values compared to IAV only and IAV + control siRNA treated samples respectively 

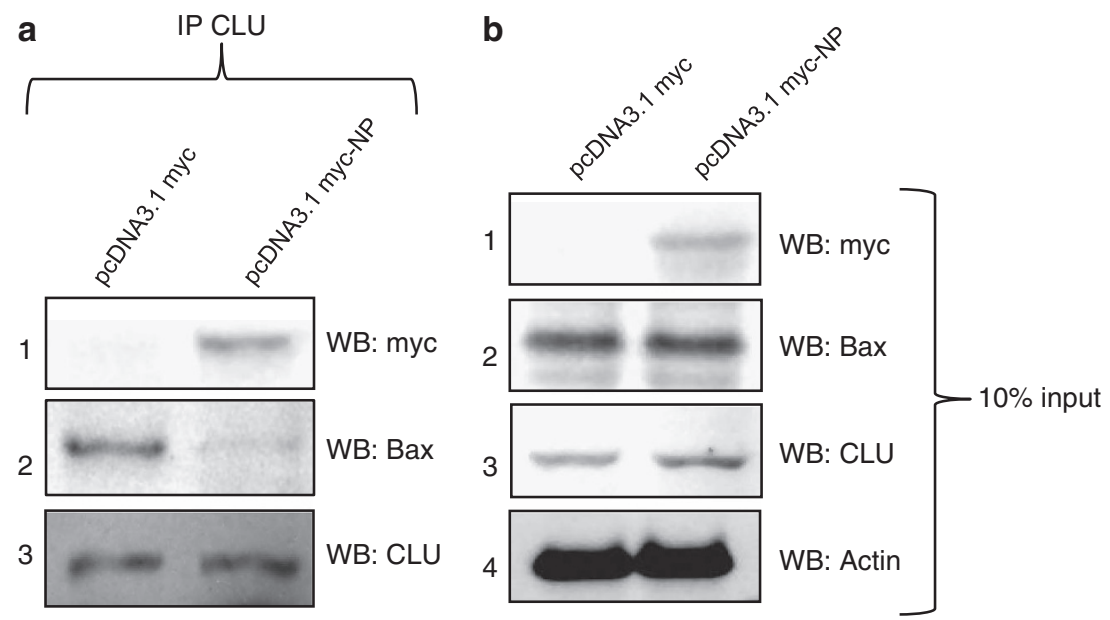

Figure 7 IAV NP expression reduces CLU-Bax association in human lung epithelial cells. A549 cells were infected with IAV NP-expressing plasmid pcDNA3.1myc-NP or control pcDNA3.1myc plasmid. Forty-eight hours post-transfection cells were harvested and immunoprecipitation was set up using CLU-specific antibody. Samples were subject to SDS-PAGE followed by western blotting with antibodies specific to myc tag and Bax, shown in panel 1 and 2 of (a). Panel 1,2, 3 and 4 of (b) show expression level of $\mathrm{NP}, \mathrm{Bax}, \mathrm{CLU}$ and $\beta$ actin in $10 \%$ of the cell lysate input used for immunoprecipitation

experiments, however, their knockdown has no effect on IAVinduced apoptosis, which is contrary to previous reports. ${ }^{6,7}$ This can be attributed to the difference in experimental conditions such as virus strains and cell type used in our study.

CLU protein prevents the initiation of intrinsic apoptotic pathway, ${ }^{23}$ and conversely IAV NP was found to activate the same. IAV NP expression was associated with Bax movement into mitochondria, release of Cyt $c$ from the mitochondria and activation of Casp 3, all of which are events involved in the intrinsic apoptosis pathway. ${ }^{30}$ Further, NP and CLU colocalized in the cytoplasm of IAV-infected cells during late stages of infection. We also observed that IAV NP-induced apoptosis in a dose-dependent manner (data not shown). Taken together, our data clearly suggest that during the late stages of the viral life cycle, when NP accumulates in the host cell, its apoptotic effect is more prominent and is mediated by the NP-CLU interaction. We also observed that CLU overexpression attenuated IAV replication in mammalian cells. It has been reported that the presence of Bax and activation of Casp 3 is essential for efficient IAV replication, ${ }^{34,35}$ and CLU is known to inhibit these molecules. ${ }^{23}$ This explains why CLU overexpression may impede IAV replication. CLU is known to interact with Bax through its $\alpha$-chain, ${ }^{23}$ whereas in the present study CLU $\beta$-chain was found to be responsible for the IAV NP-CLU interaction. Thus NP and Bax interaction sites on CLU are mutually exclusive. Moreover, we found that in the presence of NP, the CLU-Bax association was weakened in mammalian cells. Collectively, based on our data, we proposed a model for the regulation of apoptosis in IAVinfected cells by NP and propose a possible role of CLU (Figure 8). According to this model, NP interacts with CLU and either prevents its association with Bax or dissociates the Bax-CLU complex. This will lead to Bax movement into the mitochondria, release of Cyt c, activation of Casp 3 and eventually cell death. Furthermore, we found that CLU interacts exclusively with NP among IAV proteins known to promote cell death. Also the IAV NP-CLU interaction was well preserved in IAV strains of different subtypes, pathogenicity and host specificity. This suggests that IAV NP induces cell death through a specific and conserved mechanism, which is mediated by its interaction with CLU.

Few other viruses also target CLU to modulate host responses. The secreted form of CLU is exploited by the NS1 protein of Dengue viruses to interfere with the complement pathway. ${ }^{39}$ The intracellular form of CLU is known to be upregulated during Hepatitis $\delta$ virus infection, as a strategy to promote cell survival. ${ }^{40} \mathrm{CLU}$ is also known to have an important role in the regulation of PI3K signaling, NFkB pathway and cell cycle. ${ }^{41,42}$ Implications of the IAV NP interaction with CLU on these cellular processes remains to be studied. We observed that ectopic expression of CLU reduces NP or IAV-induced apoptosis, however, it does not abrogate it completely. This indicates that NP-induced cell death may not be entirely dependent on its interaction with CLU. NP is known to modulate PKR and NFkB signaling pathways, ${ }^{27,43}$ which may also have a role in NP-induced cell death.

Apoptosis induction in IAV-infected cells is closely linked to the induction of inflammatory response and viral pathogenesis. ${ }^{3}$ Exchange of gene segments between high and low pathogenic IAVs is known to alter their ability to induce cell death. ${ }^{32}$ Not surprisingly, IAV proteins such as NS1 and PB1F2, which are known to cause cytokine dysregulation, ${ }^{44-46}$ are also involved in apoptosis modulation. ${ }^{4-6} \mathrm{NP}$ is also known to interfere with the host IFN response, ${ }^{27}$ and in the current study we have shown that it contributes to host cell death also. Taken together, these facts indicate that NP could be an important virulence factor and contributor in IAV pathogenesis. It will be interesting to investigate the role of apoptotic phenotype of IAV NP in viral pathogenesis using appropriate animal models and selected IAV strains. Finally, the ubiquitous expression of CLU and the conserved nature of the IAV NP-CLU interaction makes it a promising target to develop antiviral modalities. 


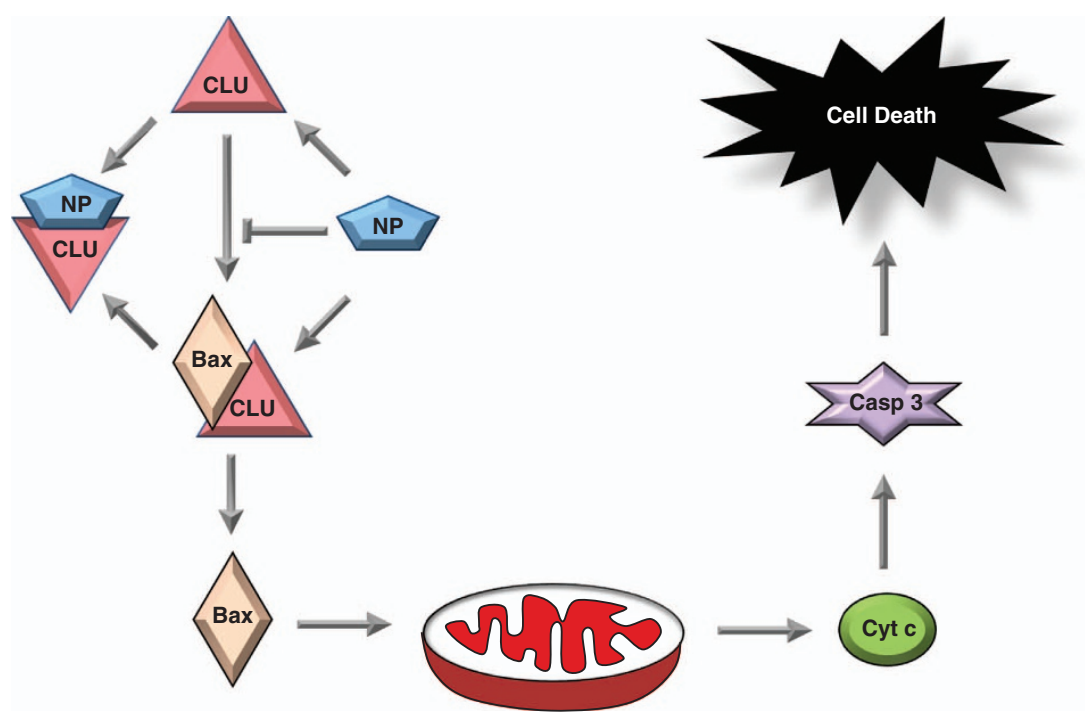

Figure 8 Model for regulation of cell death by IAV NP-CLU interaction. IAV NP interacts with human CLU protein inside the cells. Through this interaction CLU association with Bax is prevented or Clu-Bax complex is dissociated. This leads to Bax movement into the mitochondria, subsequent release of Cyt c from mitochondria, activation of Casp 3 and eventually cell death

\begin{abstract}
Materials and Methods
Cell culture and plasmids. Human lung adenocarcinoma (A549) cells were obtained from ATCC, Manassas, VA, USA and grown in DMEM medium (Hyclone, Logan, UT, USA) supplemented with 10\% fetal calf serum (FCS) (Hyclone), 100 units/ml Penicillin Streptomycin solution (Invitrogen, Grand Island, NY, USA). NHBE cells were purchased from Lonza, Basel, Switzerland and maintained as specified by supplier. The NP, NS1, M1 and M2 genes of A/Chicken/Hatay/2004 (H5N1) influenza virus were cloned in pEGFPN1 vector (Clontech, Mountain View, CA, USA). NP was also was cloned into pCDNA3.1 myc vector (Invitrogen), pGBKT7 vector (Clontech) and pET-28 $(+)$ vector (Novagen, Rockland, MA, USA). Plasmid pCMV6-XL4-CLU that expresses full-length CLU protein (NM_203339.1) was purchased from Origene (Rockville, MD, USA). For CLU domain mapping, a full-length CLU gene (without signal peptide), CLU $\beta$-chain and CLU $\alpha$-chain were cloned into pGEX4T1 vector (GE healthcare, Uppsala, Sweden) to express recombinant protein with GST tag at the N-terminus.
\end{abstract}

Transfection and IAV infection. All DNA transfections were done using Lipofectamine 2000 (Invitrogen) and cells were maintained in DMEM medium devoid of serum and antibiotics. Six hours post-transfection, culture medium was supplemented with $5 \% \mathrm{FCS}$ and $24 \mathrm{~h}$ post-transfection medium was replaced with fresh culture medium. All virus infections were done in DMEM medium supplemented with $2 \%$ BSA (GIBCO, Grand Island, NY, USA). After $1 \mathrm{~h}$ incubation with the virus, cells were washed with DMEM once and then grown in DMEM supplemented with $0.2 \% \mathrm{BSA}$ and $1 \mu \mathrm{g} / \mathrm{ml} N$-p-tosyl-1-phenyl alanine chloromethyl ketone (TPCK) (Sigma-Aldrich, St. Louis, MO, USA).

Bacterial protein expression and GST pull-down assay. The bacterial expression plasmids of IAV NP, CLU and its domains were transformed in E.coli (Rosetta strain, EMD4Biosciences, Billerica, MA, USA), and induced with $0.1 \mathrm{mM}$ IPTG overnight at $18^{\circ} \mathrm{C}$. Cells were then harvested in bacterial lysis buffer (300 mM NaCl, 50 mM NaH2PO4 pH 7.5 supplemented with $1 \mathrm{mg} / \mathrm{ml}$ lysozyme and $1 \mathrm{mM}$ PMSF). Cell suspension was sonicated and lysates were centrifuged at 13000 r.p.m. for $45 \mathrm{~min}$. Supernatants were used to setup GST pull-down assay, for which soluble fractions of GST-tagged proteins (CLU, CLU domains and GST alone as control) were incubated with GST beads (GST sepharose 4 Fast Flow, GE healthcare; no. \#17-5132-01) for $1 \mathrm{~h}$ at $4{ }^{\circ} \mathrm{C}$. Then the beads were incubated with soluble fraction containing bacterially expressed His-tagged IAV NP protein for $1 \mathrm{~h}$ at $4{ }^{\circ} \mathrm{C}$. Beads were washed three times with lysis buffer and proteinprotein complexes were collected by incubating the beads with elution buffer ( $10 \mathrm{mM}$ reduced glutathione in $50 \mathrm{mM}$ Tris-Cl pH 8.0). Eluted fractions and soluble fractions were subject to SDS-PAGE and western blotting to detect the GST or His-tagged proteins.
Western blot analyses. Cells were lysed using a buffer (20 mM HEPES, $\mathrm{pH} 7.5,150 \mathrm{mM} \mathrm{NaCl}, 1 \mathrm{mM}$ EDTA, 10\% glycerol, 1\% Triton X-100) supplemented with protease-inhibitors (Roche Diagnostics, Pleasonton, CA, USA) and the lysates were subject to SDS-PAGE. Anti-NP mouse monoclonal antibody was obtained from Immunology and Pathogenesis Branch, Influenza Division, Centers for Disease Control and Prevention, Atlanta, GA, USA. Anti-NP polyclonal antibody (ab 22285) and anti-NA antibody (ab 21304) were purchased from Abcam (Cambridge, MA, USA). Anti-NP polyclonal antibody was used specifically to detect A/California/08/2009(H1N1) NP expression. Antibodies against CLU (sc-8354, Rabbit polyclonal and sc-166907, Mouse monoclonal), IAV M1 protein (sc-69824), IAV M2 protein (sc-32238), GST tag (sc-138) and His tag (sc-803) proteins were obtained from Santa Cruz (Santa cruz, CA, USA). Anti- $\beta$-actin antibody (A2228) was purchased from Sigma-Aldrich. Anti-myc tag antibody (no. \#2276), anti-Bax antibody (no. 5023) and anti-Cytochrome c (no. \#4280) antibodies were purchased from Cell Signaling (Boston, MA, USA).

Immunoprecipitation and cellular fractionation. For coimmunoprecipitation experiments cell lysates were incubated with primary antibody overnight followed by incubation with protein A Dynabeads (Invitrogen) for $2 \mathrm{~h}$. Beads were washed three times with cold PBS. IP products were eluted by boiling the beads in SDS-PAGE sample buffer and subjected to SDS-PAGE followed by western blotting. NP was immunoprecipitated using anti-NP monoclonal antibody (Immunology and Pathogenesis Branch/IPB, CDC, Atlanta) in case of infection or anti-myc tag antibody in case of transfection. CLU was immunoprecipitated using anti-human CLU protein rabbit polyclonal antibody (sc-8354) from Santacruz Biotechnology Inc, (Santa Cruz). Biochemical fractionation of the cells was done with Qproteome cell compartment kit (Qiagen, Valencia, CA, USA, Cat. no. 37502), as per supplier's instructions.

Plaque assay. Madine Derby canine kidney cells were seeded in 6-well plates $\left(\approx 10^{6} \mathrm{cells} /\right.$ well) and the plates were incubated at $37^{\circ} \mathrm{C}$ overnight. Cell monolayers in all 6-well plates were washed twice with DMEM and the culture supernatant containing virus was added in a volume of $200 \mu \mathrm{l}$ at different dilutions. Each dilution was plated in duplicates. Plates were incubated with virus for $1 \mathrm{~h}$ followed by washing with DMEM with $0.3 \%$ BSA. The cells were overlaid with $1.6 \%$ Agarose (SeaKem LE, Cambrex, East Rutherford, NJ, USA) in L15 medium (2xL15, $1 \mathrm{M}$ HEPES, $200 \mathrm{mM}$ Glutamine, $50 \mu \mathrm{g} / \mathrm{ml}$ Gentamyin, $\mathrm{NaHCO}_{3}$ and penicillin streptomycin) with $1 \mu \mathrm{g} / \mathrm{ml}$ TPCK-treated Trypsin (Sigma-Aldrich). The plates were incubated for 2 to 3 days, agarose was removed, cells were fixed with $70 \%$ ethanol for $5 \mathrm{~min}$ and stained with crystal violet stain for $30 \mathrm{~min}$. Cells were washed with distilled water, dried and plaques were counted. 
Immunofluorescence microscopy. A549 cells infected with IAV or transfected with NP-expressing plasmid were washed with PBS, fixed in $4 \%$ paraformaldehyde for $15 \mathrm{~min}$. Cells were permeabilised with $0.5 \%$ Tween-20 in PBS for $10 \mathrm{~min}$, washed with PBST $(0.2 \%$ Tween-20), blocked with $2 \%$ BSA in PBST for $2 \mathrm{~h}$, washed with PBST, incubated with primary antibody dilution in $0.5 \%$ BSA in PBST overnight. Later cells were washed with PBST, incubated with secondary antibody (Alexa 350/405/488/594) (Invitrogen) solution in 0.5\% BSA in PBST for $2 \mathrm{~h}$ and washed with PBST. Nuclei were stained with Hoechst stain $(1 \mu \mathrm{g} / \mathrm{ml})$ (H1398), (Invitrogen) for $10 \mathrm{~min}$. For mitochondrial staining, cells were incubated with $100 \mathrm{nM}$ solution of CMXRos Red mitotracker dye (M7512 Invitrogen) in DMEM for $15 \mathrm{~min}$, followed by immunostaining. After staining was complete, cells were covered with Prolong Gold antifade mounting medium (P36930), (Invitrogen) and sides of glass cover slips were sealed. Cell images were taken under $\times 60$ objective lens of Leica DM6000B confocal microscope. Images were processed using NIS Elements AR 3.0 software (Nikon, Melville, NY, USA).

siRNA treatment. siRNAs targeting three different regions of NP, NS1, M1 and M2 genes of California/ 2009/ H1N1 IAV were designed. These siRNAs along with prevalidated siRNA against human CLU gene ${ }^{47}$ were purchased from Dharmacon (Lafayette, CO, USA). Control universal nontargeting siRNA (no. SIC001) was purchased from Sigma-Aldrich. Sequences of siRNAs used in the study are provided in the Supplementary Table 1. Transfections of siRNAs were done by using the Dharmafect 1 transfection reagent (Dharmacon). For NP/ NS1/M1/M2 silencing, A549 cells were transfected with gene-specific siRNA pool at $100 \mathrm{nM}$ and $6 \mathrm{~h}$ post-transfection, cells were infected with California/ 2009/ H1N1 virus. For CLU silencing, A549 cells were transfected with CLU siRNA at $50 \mathrm{nM}$ concentration and $24 \mathrm{~h}$ post-transfection cells were infected with California/ 2009/ H1N1 virus or transfected with NP-expressing plasmid.

Flow cytometry. Annexin V and 7 AAD staining of cells was done by using Annexin V PE apoptosis detection kit (BD Pharmingen, San Jose, CA, USA). Casp 3 activity was checked by using PE Active Caspase-3 apoptosis detection kit (BD Pharmingen). Cyt $\mathrm{c}$ release assay was performed with a FlowCellect Cyt $\mathrm{c}$ kit (Millipore, Billercia, MA, USA), in combination with PE conjugated anti-Cyt $c$ antibody from Santa Cruz (sc-13156 PE), according to the manufacturers' instructions. Samples were acquired on BD LSRII Flow cytometer (BD Biosciences) and analyzed using Flowjo version 9.3.3 software (Tree Star Inc., Ashland, OR, USA).

\section{Conflict of Interest}

The authors declare no conflict of interest.

Acknowledgements. We thank Dr. D Khang, (Institute of Biotechnology, Hanoi, Vietnam) for providing the cDNA of A/Chicken/Hatay/2004(H5N1) influenza virus. We are grateful to Dr. H Zheng (Influenza Division, CDC, Atlanta) for helping with H5N1 IAV infection experiments.Disclaimer

The findings and conclusions in this report are those of the authors and do not necessarily represent the views of the funding agency or Centers for Disease Control and Prevention.

1. Ludwig S, Pleschka S, Planz O, Wolff T. Ringing the alarm bells: signalling and apoptosis in influenza virus infected cells. Cell Microbiol 2006; 8: 375-386.

2. Everett H, McFadden G. Apoptosis: an innate immune response to virus infection. Trends Microbiol 1999; 7: 160-165

3. Brydon EWA, Morris SJ, Sweet C. Role of apoptosis and cytokines in influenza virus morbidity. FEMS Microbiol Rev 2005; 29: 837-850.

4. Chen W, Calvo PA, Malide D, Gibbs J, Schubert U, Bacik I et al. A novel influenza A virus mitochondrial protein that induces cell death. Nat Med 2001; 7: 1306-1312.

5. Schultz-Cherry S, Dybdahl-Sissoko N, Neumann G, Kawaoka Y, Hinshaw VS. Influenza Virus NS1 protein induces apoptosis in cultured cells. J Virol 2001; 75: 7875-7881.

6. Zhirnov OP, Konakova TE, Wolff T, Klenk HD. NS1 Protein of IAV down-regulates apoptosis. J Virol 2002; 76: 1617-1625.

7. Halder UC, Bagchi P, Chattopadhyay S, Dutta D, Chawla-Sarkar M. Cell death regulation during influenza A virus infection by matrix (M1) protein: a model of viral control over the cellular survival pathway. Cell Death Dis 2011; 2: e197.

8. Rossman JS, Lamb RA. Autophagy, apoptosis, and the Influenza virus M2 protein. Cell Host Microbe 2009; 6: 299-300.
9. Carlson CM, Turpin EA, Moser LA, O'Brien KB, Cline TD, Jones JC et al. Transforming growth factor- $\beta$ : activation by neuraminidase and role in highly pathogenic $\mathrm{H} 5 \mathrm{~N} 1$ influenza pathogenesis. PLoS Pathog 2010; 6: e1001136.

10. Gaur P, Ranjan P, Sharma S, Patel JR, Bowzard JB, Rahman SK et al. Influenza A virus neuraminidase protein enhances cell survival through interaction with carcinoembryonic antigen-related cell adhesion molecule 6 (CEACAM6) protein. J Biol Chem 2012; 18: 15109-15117

11. Tada T, Suzuki K, Sakurai $\mathrm{Y}$, Kubo M, Okada H, Itoh T et al. NP body domain and pb2 contribute to increased virulence of $\mathrm{H} 5 \mathrm{~N} 1$ highly pathogenic avian influenza viruses in chickens. J Virol 2011; 85: 1834-1846.

12. Gabriel G, Herwig A, Klenk HD. Interaction of polymerase subunit PB2 and NP with importin alpha1 is a determinant of host range of influenza A virus. PLoS Pathog 2008; 4: e11.

13. Portela A, Digard P. The influenza virus nucleoprotein: a multifunctional RNA-binding protein pivotal to virus replication. J Gen Virol 2002; 83: 723-734.

14. Ye Q, Krug RM, Tao YJ. The mechanism by which influenza $A$ virus nucleoprotein forms oligomers and binds RNA. Nature 2006; 444: 1078-1082.

15. Biswas SK, Boutz PL, Nayak DP. Influenza virus nucleoprotein interacts with influenza virus polymerase proteins. J Virol 1998; 72: 5493-5501.

16. Noton SL, Medcalf E, Fisher D, Mullin AE, Elton D, Digard P. Identification of the domains of the influenza A virus M1 matrix protein required for NP binding, oligomerization and incorporation into virions. J Gen Virol 2007: 88: 2280-2290.

17. Robb NC, Chase G, Bier K, Vreede FT, Shaw PC, Naffakh N et al. The influenza A virus NS1 protein interacts with the nucleoprotein of viral ribonucleoprotein complexes. J Virol 2011; 85: 5228-5231.

18. Elton D, Simpson-Holley M, Archer K, Medcalf L, Hallam R, McCauley J et al. Interaction of the influenza virus nucleoprotein with the cellular CRM1-mediated nuclear export pathway. $J$ Virol 2001; 75: 408-419.

19. Digard P, Elton D, Bishop K, Medcalf E, Weeds A, Pope B. Modulation of nuclear localization of the influenza virus nucleoprotein through interaction with actin filaments. J Virol 1999; 73: 2222-2231.

20. Momose F, Basler CF, O'Neill RE, Iwamatsu A, Palese P, Nagata K. Cellular splicing factor RAF-2p48/NPI-5/BAT1/UAP56 interacts with the influenza virus nucleoprotein and enhances viral RNA synthesis. J Virol 2001; 75: 1899-1908.

21. Galluzzi L, Brenner C, Morselli E, Touat Z, Kroemer G. Viral control of mitochondrial apoptosis. PLoS Pathog 2008; 4: e1000018.

22. Trougakos IP, Gonos ES. Clusterin/Apolipoprotein $J$ in human aging and cancer. Int J Biochem Cell Biol 2002; 34: 1430-1448.

23. Zhang H, Kim JK, Edwards CA, Xu Z, Taichman R, Wang CY. Clusterin inhibits apoptosis by interacting with activated Bax. Nat Cell Biol 2005; 7: 909-915.

24. Hai R, Schmolke M, Varga ZT, Manicassamy B, Wang TT, Belser JA et al. PB1-F2 expression by the 2009 pandemic $\mathrm{H} 1 \mathrm{~N} 1$ influenza virus has minimal impact on virulence in animal models. J Virol 2010; 84: 4442-4450.

25. Vermes I, Haanen C, Steffens-Nakken H, Reutelingsperger C. A novel assay for apoptosis. Flow cytometric detection of phosphatidylserine expression on early apoptotic cells using fluorescein labelled Annexin V. J Immunol Methods 1995; 184: 39-51.

26. Lecoeur H, de Oliveira-Pinto LM, Gougeon ML. Multiparametric flow cytometric analysis of biochemical and functional events associated with apoptosis and oncosis using the 7-aminoactinomycin D assay. J Immunol Methods 2002; 265: 81-96.

27. Sharma K, Tripathi S, Ranjan P, Kumar P, Garten R, Deyde V et al. Influenza A virus nucleoprotein exploits Hsp40 to inhibit PKR activation. PLoS One 2011; 6: e20215.

28. Burkey BE, deSilva HV, Harmony JA. Intracellular processing of apolipoprotein J precursor to the mature heterodimer. J Lipid Res 1991; 32: 1039-1048.

29. Leskov KS, Araki S, Lavik JP, Gomez JA, Gama V, Gonos ES et al. CRM1 proteinmediated regulation of nuclear clusterin (nCLU), an ionizing radiation-stimulated, Baxdependent pro-death factor. J Biol Chem 2011; 46: 40083-40090

30. Mayer B, Oberbauer R. Mitochondrial regulation of apoptosis. News Physiol Sci 2003; 18 : 89-94.

31. Hoetelmans R, van Slooten HJ, Keijzer R, Erkeland S, van de Velde CJ, Dierendonck JH. $\mathrm{Bcl}-2$ and Bax proteins are present in interphase nuclei of mammalian cells. Cell Death Differ 2000; 7: 384-392.

32. Morris SJ, Nightingale K, Smith $\mathrm{H}$, Sweet C. IAV-induced apoptosis is a multifactorial process: exploiting reverse genetics to elucidate the role of IAV proteins in virus-induced apoptosis. Virology 2005; 335: 198-211.

33. Olsen CW, Kehren JC, Dybdahl-Sissoko NR, Hinshaw VS. Bcl-2 alters influenza virus yield, spread, and hemagglutinin glycosylation. J Virol 1996; 70: 663-666.

34. McLean JE, Datan E, Matassov D, Zakeri ZF. Lack of Bax prevents IAV-induced apoptosis and causes diminished viral replication. J Virol 2009; 83: 8233-8246.

35. Wurzer WJ, Planz O, Ehrhardt C, Giner M, Silberzahn T, Pleschka S et al. Caspase 3 activation is essential for efficient influenza virus propagation. EMBO J 2003; 22: 2717-2728.

36. Zhirnov OP, Konakova TE, Garten W, Klenk HD. Caspase-dependent N-terminal cleavage of influenza virus nucleocapsid protein in infected cells. J Virol 1999; 73: 10158-10163.

37. Coleman JR. The PB1-F2 protein of influenza A virus: increasing pathogenicity by disrupting alveolar macrophages. Virol J 2007; $4: 9$.

38. Korteweg C, Gu J. Pathology, molecular biology, and pathogenesis of avian influenza A (H5N1) infection in humans. Am J Pathol 2008; 172: 1155-1170. 
39. Kurosu T, Chaichana P, Yamate M, Anantapreecha S, Ikuta K. Secreted complement regulatory protein clusterin interacts with dengue virus nonstructural protein1. Biochem Biophys Res Commun 2007; 362: 1051-1056.

40. Liao FT, Lee YJ, Ko JL, Tsai CC, Tseng CJ, Sheu GT. Hepatitis delta virus epigenetically enhances clusterin expression via histone acetylation in human hepatocellular carcinoma cells. J Gen Virol 2009; 90: 1124-1134.

41. Ammar $\mathrm{H}$, Closset JL. Clusterin activates survival through the phosphatidylinositol 3-kinase/Akt pathway. J Biol Chem 2008; 283: 12851-12861.

42. Santilll G, Aronow BJ, Sala A. Essential requirement of apolipoprotein J (Clusterin) signaling for I_B expression and regulation of NF-kB ACTIVITY. J Biol Chem 2003; 278 : 38214-38219.

43. Flory E, Kunz M, Scheller C, Jassoy C, Stauber R, Rapp UR et al. Influenza virus-induced NF-kappaB-dependent gene expression is mediated by overexpression of viral proteins and involves oxidative radicals and activation of IkappaB kinase. J Biol Chem 2000; 275 : 8307-8314.

44. Zamarin D, Ortigoza MB, Palese P. Influenza A virus PB1-F2 protein contributes to viral pathogenesis in mice. J Virol 2006; 80: 7976-7983.
45. Varga ZT, Ramos I, Hai R, Schmolke M, García-Sastre A, Fernandez-Sesma A et al. The influenza virus protein PB1-F2 inhibits the induction of type I interferon at the level of the MAVS adaptor protein. PLoS Pathog 2011; 7: e1002067.

46. Kochs G, García-Sastre A, Martínez-Sobrido L. Multiple anti-interferon actions of the influenza A virus NS1 protein. J Virol 2007; 81: 7011-7021.

47. Trougakos IP, So A Jansen B, Gleave ME, Gonos ES. Silencing expression of the clusterin/apolipoprotein $\mathrm{J}$ gene in human cancer cells using small interfering RNA induces spontaneous apoptosis, reduced growth ability, and cell sensitization to genotoxic and oxidative stress. Cancer Res 2004; 64: 1834-1842.

(c) (i) (2) Cell Death and Disease is an open-access journal published by Nature Publishing Group. This work is licensed under a Creative Commons Attribution-NonCommercialShareAlike 3.0 Unported License. To view a copy of this license, visit http://creativecommons.org/licenses/by-nc-sa/3.0/

Supplementary Information accompanies this paper on Cell Death and Disease website (http://www.nature.com/cddis) 